\title{
A New Method for Finding the Shoulder Complex Rotation Centre Using 3D Body Scanning
}

\author{
S.G. Rozevink', I. Kingma', D. Spies², M.I. Paalman², J. Molenbroek ${ }^{3}$, \\ B. Naagen ${ }^{3}$, M. Maas ${ }^{4}$, G.Streekstra ${ }^{4}$, H.A.M. Daanen ${ }^{* 1}$ \\ ${ }^{1}$ Faculty of Behavioural and Movement Sciences, Vrije Universiteit Amsterdam, The Netherlands; \\ ${ }^{2}$ Yumen Bionics, Amsterdam, The Netherlands; \\ ${ }^{3}$ Industrial Design Engineering, Technical University Delft, Delft, The Netherlands; \\ ${ }^{4}$ Dept. of Radiology \& Nuclear Medicine, Division of Musculoskeletal Radiology, \\ University of Amsterdam, Academic Medical Center, Amsterdam, The Netherlands
}

DOI: 10.15221/18.019 http://dx.doi.org/10.15221/18.019

\begin{abstract}
To avoid radiation exposure to the body, the rotation centre of the shoulder complex is often assessed using the Optotrak motion capture system instead of X-ray imaging. Recently, 3D body scanning techniques evolved from static to temporal scanning. This study investigated if a time series of $3 \mathrm{D}$ body scans may be an alternative for motion capture systems focussing on the shoulder complex rotation centre which is relevant for exoskeleton alignment to the user's body. 13 Male participants participated in this study (age $24.8 \pm 2.4$ years, stature $182.2 \pm 5.5 \mathrm{~cm}$, body mass $80.7 \pm 7.3 \mathrm{~kg}$ ). Motion capture data (Optotrak) and 3D body scans (3dMD) were collected while the participant performed predefined movements (abduction, anteflexion and a combination of the two). The helical axes method was used to calculate the rotation centre from motion capture data as reference. The 3D scans were processed modelling the upper arm as a cylinder or as a set of perpendicular slices to the centroid axis. Also, a point 30 millimetres below the acromioclavicular joint, assessed from the 3D scan, was defined as the conventional method. The mean distance from the rotation centre of each individual to the mean rotation centre of the cylinder $(19 \mathrm{~mm})$, centroid $(18 \mathrm{~mm})$, conventional $(16$ $\mathrm{mm}$ ) and helical axis method $(21 \mathrm{~mm})$ were much smaller than the distances between the 3D scan derived methods and the helical axes method (43-49 mm). The shoulder complex rotation centre location differed considerably between the four methods. Therefore, X-rays of the shoulder are necessary as a golden standard to indicate which method is closest to the real rotation centre and to determine the correction factor for each method that removes the systematic error. 3D body scans contain valuable anthropometric information and have the potential for biomechanical modelling since the random error does not exceed the error of the Optotrak motion capture system.
\end{abstract}

Keywords: 3D body scanning, exoskeleton, Duchenne, rotation centre, shoulder complex

\section{Introduction}

The human shoulder is a complex structure since it consists of multiple joints. There is no consensus on how to correctly model the shoulder. It can be modelled as a rotation point or as a rotation axis [1], [2]. Moreover, the shoulder can be seen as a complex or as individual joints [3], [4]. The golden standard is to estimate a joint rotation centre using X-rays or computed tomography (CT) scans[5][9]. Since the shoulder complex is not one individual joint and to avoid unnecessary radiation exposure, it is recommended to replace this invasive method by an alternative approach such as motion capture [8], [10]-[17]. The traditional way is to use helical axes (HA) to calculate the rotation centre of the shoulder complex, this method is validated for the glenohumeral joint [8], [10], [20], [11][16], [18], [19]. It takes a considerable amount of time to collect motion capture data and the motion capture system (Optotrak) is very expensive. To overcome these problems, we investigated if 3D body scanning can be used to calculate the rotation centre of the shoulder complex. 3D scanning is an upcoming way of measuring shapes and sizes of objects and humans for several applications [21], [22]. Recently, 3D body scan technology improved in such a way that several scans per second can be made. This opens the potential for time series analysis of 3D scans. This technology may offer an alternative for X-ray or the expensive Optotrak system.

*h.a.m.daanen@vu.nl, +31(0)20-5982481; www.vu.nl 
Furthermore, individual variables such as anthropometric data can easily be extracted from the model. Differences in anatomy between humans underline the importance for the individual determination of the shoulder complex rotation centre. Van der Helm et al. (1992) compared different cadaveric arms for musculoskeletal modelling [4]. They concluded that the intra-individual differences were occasionally as large as the inter-individual differences. The individual data per specimen was not displayed, hence it cannot be determine what specific differences were present between the cadaveric bodies. They state that it was not possible to average over the cadavers. Therefore, we emphasize the need for individual determination of the rotation centre. An applied example of the necessity to know the individual shoulder complex rotation centre is the design of upper body exoskeletons. We want to contribute to the development of an existing passive exoskeleton; the Agear [23], [24]. The movements of this exoskeleton are designed around a virtual rotation point, which is captured in a gimbal joint which can move in three directions. This gimbal joint represents the shoulder complex and its location is the most important parameter for alignment to the user. A group of patients who can benefit from these upper arm orthoses are Duchenne patients. Duchenne muscular dystrophy is a progressive disease which affects 4.78 per 100,000 males [25]. This disease is characterized by weakness which spreads progressively from proximal to distal. Assisted by the exoskeleton, a patient can retain some of his independence. The A-gear is lightweight and less conspicuous than other designs. The device consists of rigid links, elastic bands, hinge joints, polymer pads and a lower arm support. By a specific configuration of the elastic spring elements, the weight of the user's arm is balanced over the entire range of motion [26]. One of the challenges for exoskeletons is the precise alignment to the user for optimal performance. During the development of the A-gear exoskeleton, the rotation centre was chosen to be located 30 millimetres $(\mathrm{mm})$ below the acromioclavicular (AC) joint, this will be called the conventional method. We speculate that there is room for improvement to locate this point individually for better alignment. Furthermore, individual anthropometric data for the alignment is currently measured using callipers and measurement tape. The 3D body scans contain precious information about the form, distances and depth of the body. This would make a separate conventional measurement session with measurement tape unnecessary. The purpose of this thesis was to investigate the potential of using the new challenging technology of 3D body scanning to estimate the shoulder complex rotation centre. More specifically, our goal is to locate the individual rotation centre of the shoulder complex in a thorax coordinate system for a better alignment of the exoskeleton using 3D body scanning. We strive to make the algorithm quick and easy to use by automating the process. This would make it feasible to use this method outside of the current research scope, perhaps for other joints. It was hypothesized that: 1) calculation of the shoulder complex rotation centre using 3D body scan methods obtain the same result as calculated from the HA method; 2) 3D body scan methods result in an estimate of the rotation centre closer to the HA method in comparison to the conventional method.

\section{Methods}

\subsection{Ethics}

The ethical committee of the Vrije Universiteit of Amsterdam approved the protocol for this study. Before the measurements, the participants were informed about the procedures and signed a written informed consent.

\subsection{Participants}

In this study, 13 healthy male subjects between the age of 20 and 30 years old (24.8 years \pm 2.4 ) participated. They had no shoulder complaints in the last six months. Their body dimensions are representative of the Dutch population (Central Bureau of Statistics: stature $=183.2 \mathrm{~cm}$, body mass $=80.4 \mathrm{~kg}$ [27]). The characteristics of the participants are presented in Table 1. Healthy male participants were studied to prevent unnecessary stress for the Duchenne patients in the current stage of investigation. 
Table 1. Characteristics of the 13 male participants. The mean and standard deviation (SD) are presented for age (year), body mass (kilogram), stature (centimetre) and body mass index (BMI, kilogram/metre ${ }^{2}$ ).

\begin{tabular}{ccc}
\hline & Mean & SD \\
\hline Age $(\mathrm{y})$ & 24.8 & 2.4 \\
Body mass $(\mathrm{kg})$ & 80.7 & 7.3 \\
Stature $(\mathrm{cm})$ & 182.2 & 5.5 \\
BMI $\left(\mathrm{kg} / \mathrm{m}^{2}\right)$ & 24.3 & 1.5
\end{tabular}

\subsection{Data acquisition}

Nine specific marker points were incorporated in our point cloud model (see figure 1). The following palpable bony landmarks were marked with kohl pencil on the bare upper body. The suprasternal notch (ST), xiphoid process (XP), 7th cervical vertebrae (C7) and the 6th thoracic vertebrae (T6) were palpated to construct a thoracic coordinate system. The AC joint was located to determine the rotation centre based on the conventional method. The angulus acromion (AA) was included to obtain a complete 3D model using motion capture. The cubital fossa (CF), lateral epicondyle (EL) and medial epicondyle (EM) were marked to calculate a mid-centre of the elbow.
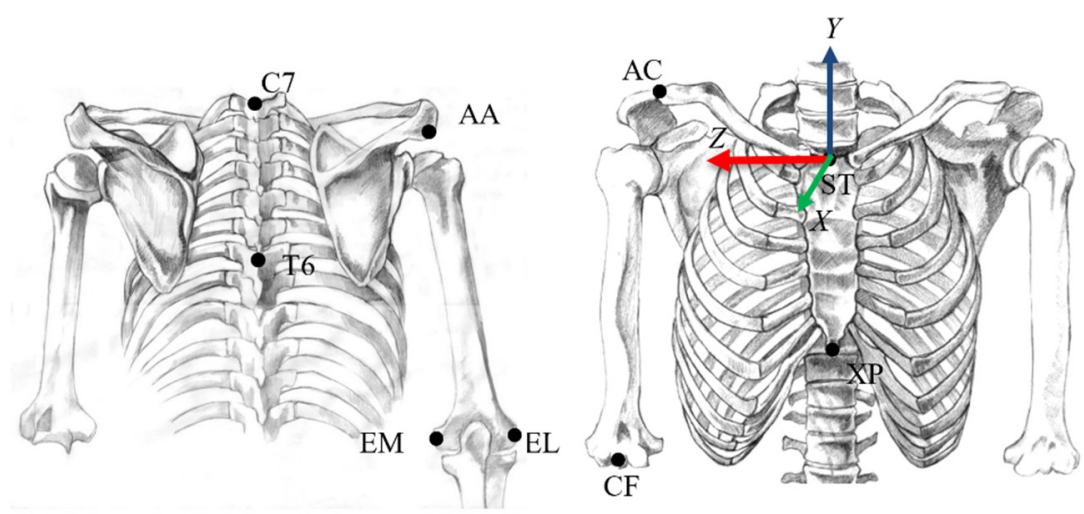

Fig. 1. Visual representation of the palpable bony landmarks which were marked on the bare upper body of the participant. The $7^{\text {th }}$ cervical vertebrae (C7), $6^{\text {th }}$ thoracic vertebrae (T6), angulus acromion (AA), lateral epicondyle

(EL) and medial epicondyle (EM) were marked on the dorsal side of the body. The suprasternal notch (ST),

xiphoid process $(X P)$, acromioclavicular joint $(A C)$, cubital fossa $(C F)$ were marked on the ventral side of the body. The coloured arrows represent the coordinate system ( $X=$ green, $Y=$ blue, $Z=$ red) which is located in the suprasternal notch.

\subsubsection{D body scan}

The participant performed a series of movements in the 3D body scanner at Delft University (3dMDbody.t system, Atlanta, Georgia, USA). The protocol was adapted from a movement protocol to determine the reachable workspace [28]. The participant started from a basic position; the right arm was positioned passively beside the body. He made an abduction to $90^{\circ}$, then returned to the basic position. Subsequently, a diagonal anteflexion to $90^{\circ}$ was performed, i.e. an anteflexion motion performed at $45^{\circ}$ horizontal abduction. The last part of the motion was $90^{\circ}$ anteflexion. To perform the movement fluently and with the right velocity, a metronome with 84 beats per minute was used and the participant was instructed to use six taps to perform one motion (three taps upward motion, three taps downward motion). The motion was practised a few times before the actual measurement was started. Furthermore, the participant was asked to move only the arm and not the whole shoulder complex to avoid unnecessary elevation of the shoulder.3D scans were processed by the software of $3 \mathrm{dMD}$ to point cloud models. Since this scanner is able to record 10 3D scans per second, specific postures at $20^{\circ}, 55^{\circ}$ and $90^{\circ}$ of the motions were selected afterwards from the time series using CloudCompare [29]. Figure 2 shows the extracted point clouds in frontal and transverse view. 


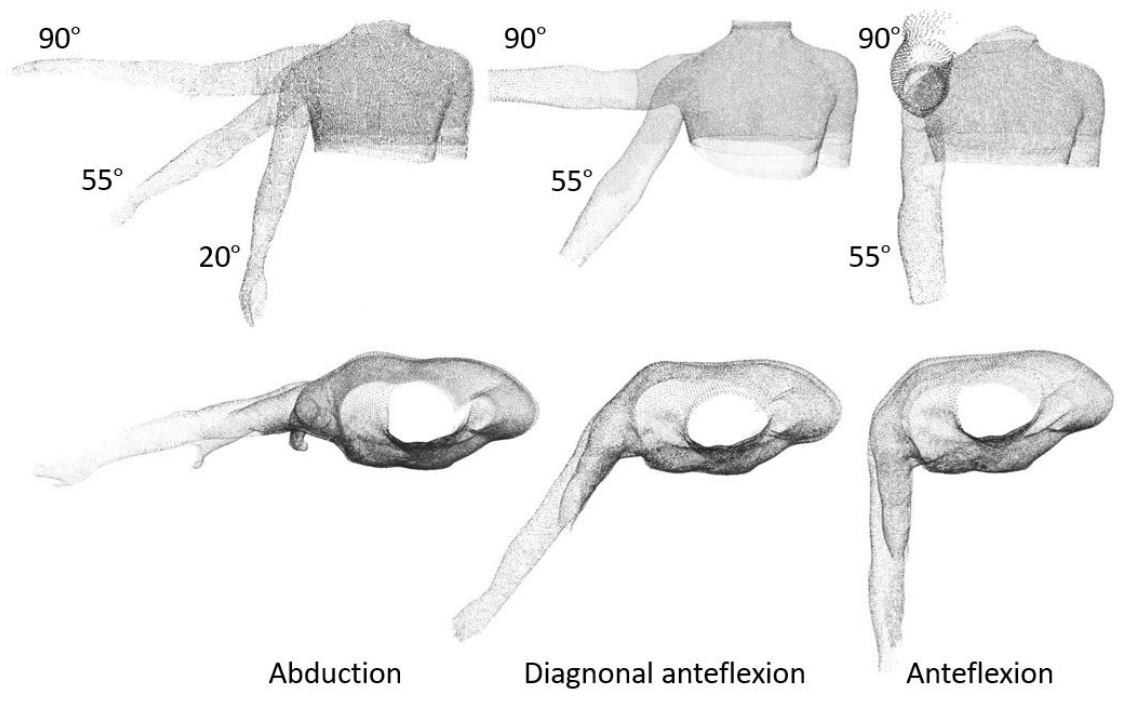

Fig. 2. The postures for analysis were afterwards extracted from the time series of point clouds. The upper row shows the seven point clouds of PP_003 in frontal view, note the three levels of elevation $\left(20^{\circ}, 55^{\circ}\right.$ and $\left.90^{\circ}\right)$. In the lower row, the same point clouds are presented but viewed from above, this transverse view clearly shows the three performed motions: abduction, diagonal anteflexion (anteflexion at $45^{\circ}$ horizontal abduction) and anteflexion.

\subsubsection{Helical axes}

Five months later, motion recordings were performed using two Optotrak beams (Vrije Universiteit, Amsterdam). Data were collected at a sample rate of $100 \mathrm{~Hz}$. The same bony landmarks were pointered equal to the 3D body scan. The movement protocol was separated in three consecutive trials of an abduction, diagonal anteflexion and anteflexion to $90^{\circ}$. Every trial had a duration of $20 \mathrm{~s}$ and every motion was repeated 7 or 8 times. A metronome was not used because the motion should be performed fluently, the beat of the metronome could result in a jerky movement which was undesired since the helical axes (HA) calculation is dependent on the velocity.

\subsection{Data analysis}

\subsubsection{D body scan}

The point clouds of the selected postures were opened in Meshlab where the pick point tool was used to extract the bony landmarks [30]. Subsequently, the point clouds were opened in Geomagic [31]. This program contains a smart tool which can create a bridge between two points, this was useful for filling holes in the armpit due to occlusion. By first creating a bridge, the hole in the arm and trunk can be filled more precise according to the shape of the armpit. After filling holes, two point clouds were saved per posture: one of the whole upper body (minus the head) and one with only the cylindrical part of the right upper arm (perpendicular to centroid axis between the armpit and the midpoint of the two epicondyles). The point clouds and marker points were loaded into Matlab (2017). They were translated and rotated into a thorax coordinate system [20]. We used the 6th thoracic vertebrae instead of the 8th vertebrae since this was convenient for the motion capture measurements. This could result in a negligible difference in the direction of the Y-axis. In accordance to Wu et al. (2005), the origin is in the ST, the X-axis is pointing ventral, Y-axis upwards and Z-axis lateral to the right (Eq.1-4, see figure 1) [20]. Subsequently, the point cloud and marker points were transformed to the thorax coordinate system (Eq.5)

$$
\begin{gathered}
Y_{\text {thorax }}=\frac{C 7+S T}{2}-\frac{T 6+X P}{2} \\
Z_{\text {thorax }}=\left(S T-\frac{C 7+S T}{2}\right) \times Y_{\text {thorax }}
\end{gathered}
$$




$$
\begin{gathered}
X_{\text {thorax }}=Y_{\text {thorax }} \times Z_{\text {thorax }} \\
R_{\text {thorax }}=\left[X_{\text {thorax }} Y_{\text {thorax }} Z_{\text {thorax }}\right] \\
\text { Point cloud }_{\text {thorax }}=\operatorname{inv}\left(R_{\text {thorax }}\right) \cdot\left(\text { Point cloud } \text { global }_{\text {toinal }}-S T_{\text {global }}\right)
\end{gathered}
$$

The bony landmarks on the elbow (both epicondyle and cubital fossa) were averaged to find the midpoint of the elbow. A reference vector $(\overrightarrow{R V})$ was created using the rotation centre calculated via the conventional method $\left(\overrightarrow{R C_{Y B}}\right)$ to the midpoint of the elbow (Eq.6).

$$
\overrightarrow{R V}=\overrightarrow{R C_{Y B}}-\frac{\overrightarrow{E L}+\overrightarrow{E M}+\overrightarrow{C F}}{3}
$$

This reference vector was used as an initial guess in the model to specify the direction of the fitted cylinders. The humeral shaft can be represented by a cylindrical shape and a cylinder is roughly the best estimate of the shape of the upper arm [4], [32]-[34]. The cylindrical part of the upper arm (i.e. from elbow to armpit) was used to fit a cylinder based on a random sampling method [35] (see figure 3). The cylinder fit function is integrated in Matlab (2015 and higher), for underlying functions researchers are referred to the article of Torr et al. (2000) [35].

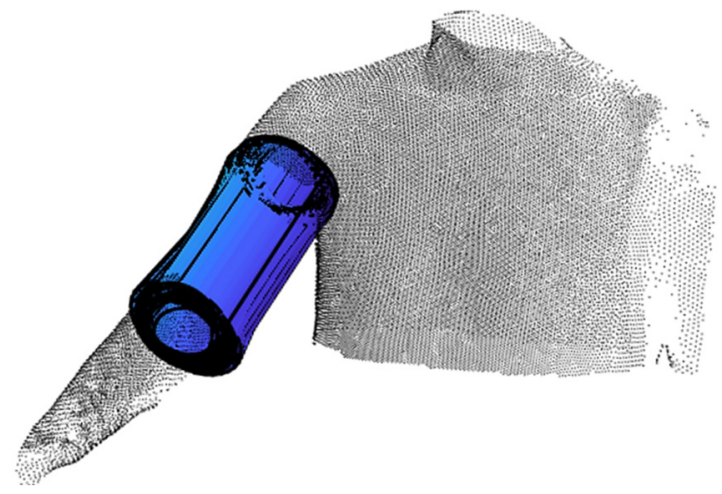

Fig. 3. In the cylinder method, a cylindrical shape was fitted through the point cloud of the upper arm between the midpoint of the elbow and a point $30 \mathrm{~mm}$ caudal of the acromioclavicular joint.

This procedure of cylinder fitting was performed 250 times per point cloud to provide a robust estimate. Every time a cylinder could be fitted through the point cloud with $99 \%$ confidence, a midline of the cylinder was calculated $\left(L_{i}\right)$. For every posture, one mean mid axis $(\bar{L})$ was calculated, with $n$ being the amount of fitted cylinders (Eq.7).

$$
\bar{L}=\frac{1}{n} \sum_{i=1}^{n} L_{i}
$$

For each posture the mean line $(\bar{L})$ was obtained which runs through the upper arm and shoulder joint. The seven postures of one participant were combined. Since we are working in three dimensions, it was very unlikely that the mid lines would cross each other. Therefore, the shortest distances $(d)$ between all possible combinations of postures were calculated (Eq.8-10). In these equations, $L_{j}$ and $L_{k}$ are a pair of lines that ought to be compared and a and $\mathrm{b}$ are the parameters of each line.

$$
\begin{aligned}
& L_{j}: \vec{r}=\overrightarrow{a_{1}}+\lambda \overrightarrow{b_{1}} \\
& L_{k}: \vec{r}=\overrightarrow{a_{2}}+\lambda \overrightarrow{b_{2}}
\end{aligned}
$$




$$
d=\frac{\left|\left(\overrightarrow{a_{2}}-\overrightarrow{a_{1}}\right) \cdot\left(\overrightarrow{b_{1}} \times \overrightarrow{b_{2}}\right)\right|}{\left|\overrightarrow{b_{1}} \times \overrightarrow{b_{2}}\right|}
$$

Every shortest distance line $(d)$ had an intersecting point on both lines $\left(P_{1}\right.$ and $P_{2}$, Eq.13-14), the mean of the shortest distance line (point $M$ ) was calculated by taking the mean of these two points (Eq.15).

$$
\begin{gathered}
\overrightarrow{n_{1}}=\overrightarrow{b_{1}} \times \overrightarrow{b_{2}} \\
\overrightarrow{n_{2}}=\overrightarrow{b_{2}} \times \overrightarrow{n_{1}} \\
\overrightarrow{P_{1}}=\overrightarrow{a_{1}}+\frac{\left(\overrightarrow{a_{2}}-\overrightarrow{a_{1}}\right) \cdot \overrightarrow{n_{2}}}{\overrightarrow{b_{1}} \cdot \overrightarrow{n_{2}}} \\
\overrightarrow{P_{2}}=\overrightarrow{a_{2}}+\frac{\left(\overrightarrow{a_{1}}-\overrightarrow{a_{2}}\right) \cdot \overrightarrow{n_{1}}}{\overrightarrow{b_{2}} \cdot \overrightarrow{n_{1}}} \\
{\overrightarrow{m_{i}}}_{i}=\frac{\overrightarrow{P_{1}}+\overrightarrow{P_{2}}}{2}
\end{gathered}
$$

The average value of all the midpoints resulted in our estimate of a rotation centre (Eq.16).

$$
\text { Rotation centre }=\overrightarrow{\vec{M}}=\frac{1}{n} \sum_{i=1}^{n} \vec{M}_{i}
$$

The second method was slightly different from the first method. In the centroid method, the point cloud of the upper arm (i.e. from armpit to elbow) was sectioned into perpendicular slices to give a 2D representation of the contour of the upper arm. A 3D circle was fitted through a contour slice by a least square fit using Gauss-Newton algorithm (see figure 4). Assuming that the humeral bone lies perfectly in the middle of the arm, we calculated the centroid of this circle and state that this is a point on the humerus midline. If a straight line was fitted through the centroids, this would represent the location of the bone.

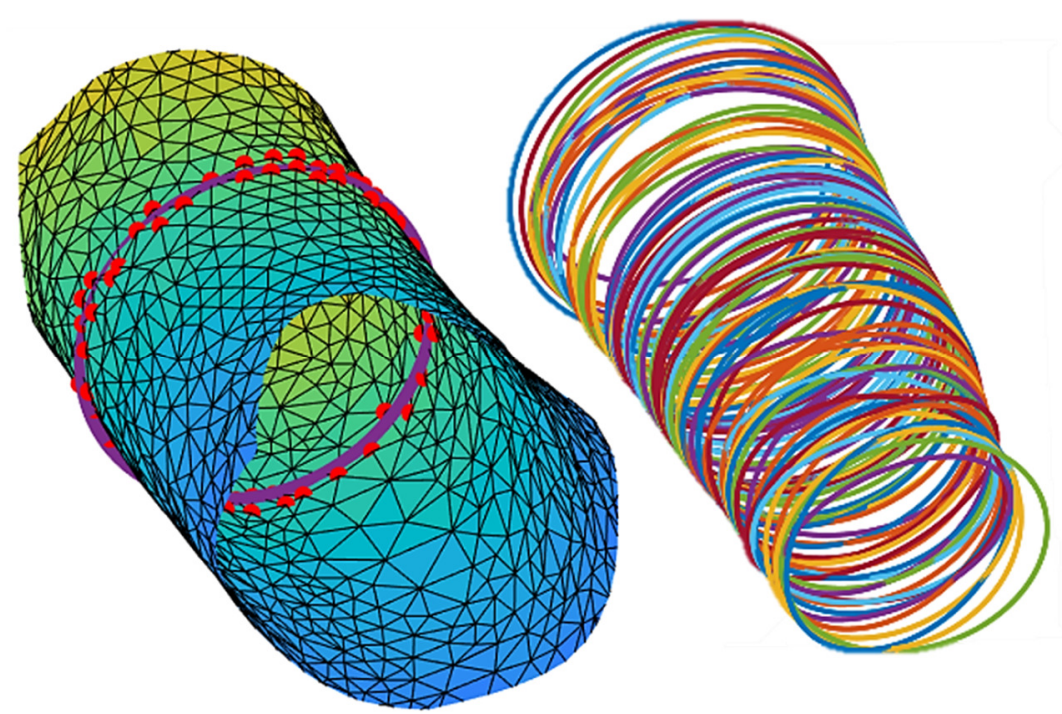

Fig. 4. In the centroid method, perpendicular slices of the upper arm were used to fit circles. On the left, a triangulated mesh is depicted where one circle (purple) is fitted through a perpendicular slice of the point cloud (red dots). On the right, all circles are fitted through the point cloud.

The fitted line was subsequently used to estimate the rotation centre in the same way as the mid-axis line of the fitted cylinders was used in the first method.

Currently, the rotation centre is estimated as $30 \mathrm{~mm}$ below the $A C$ joint, therefore a point $30 \mathrm{~mm}$ caudal of the AC was considered as an estimate for the conventional method. 


\subsubsection{D body scan}

The data were interpolated and filtered using a 4th order low-pass digital Butterworth filter with a cutoff frequency of $5 \mathrm{~Hz}$. The data were translated and rotated to our thorax coordinate system (Eq. 1-5). The trajectory of the position of the humerus with respect to the thorax was used to calculate the HA of that motion. The HA is a directed line where a rigid body can rotate around while also translate along that line [36]. During a movement, the instantaneous HA is different for every moment in time. For every instant, the direction vector $(n)$ and the position vector of the axis $(s)$ were calculated using the position $(p)$ and velocity $(\dot{p})$ of the humerus with respect to the thorax and the angular velocity vector $(\omega)$ (Eq. 19-20). The angular velocity was calculated using the rotation matrix $(R)$ and the numerical derivative $(\dot{R})$ of the upper arm with respect to the thorax (Eq. 17-18).

$$
\begin{gathered}
\omega_{\text {temp,i }}=\frac{\dot{\mathrm{R}}_{\mathrm{i}} \cdot \mathrm{R}_{\mathrm{i}}^{\mathrm{T}}-\mathrm{R}_{\mathrm{i}} \cdot \dot{\mathrm{R}}_{\mathrm{i}}^{\mathrm{T}}}{2} \\
\omega_{\mathrm{i}}=\omega_{\text {temp,i }}(3,2) \\
\omega_{\text {temp, }, \mathrm{i}}(2,3) \\
\mathrm{n}_{\mathrm{i}}=\frac{\omega_{\mathrm{i}}}{\left\|\omega_{\mathrm{i}}\right\|} \\
\mathrm{s}_{\mathrm{i}}=\mathrm{p}_{\mathrm{i}}+\omega_{\mathrm{i}} \times \frac{\dot{\mathrm{p}}_{\mathrm{i}}}{\left\|\omega_{\mathrm{i}}\right\|^{2}}
\end{gathered}
$$

All the direction vectors and position vectors were used to estimate the optimal $\mathrm{HA}$ and pivot point by minimizing the distance between the instantaneous HA according to Woltring [37].

\subsubsection{Statistics}

From our measurements, the coordinates of the rotation centre were obtained in a thorax coordinate system, resulting in four rotation centre estimates per participant, expressed in X-, Y- and Zcoordinates. This study had a repeated measures design, which means that the same parameter was measured multiple times using different methods. A repeated measures analysis of variance (ANOVA) was used to test if there were significant differences between the coordinates of the rotation centre obtained from the motion capture data in comparison to the three 3D body scan derived methods. The location is expressed in three dimensions: X-, Y- and Z-coordinates, each axis was tested separately. Alpha is set to 0.05. A pairwise comparison with Bonferroni correction showed which methods were significantly different. A non-significant difference between the location of a 3D scan method and HA method proves that the methods result in a similar location of the rotation centre.

\section{Results}

The rotation centres of the cylinder, centroid and conventional method were compared to the HA method per X-, Y- and Z-coordinate. The results showed that all methods were significantly different from each other in all directions $(p<0.01)$. Post hoc tests showed that the difference between the $X-$ coordinate of the conventional method and the HA method and the difference between the Zcoordinate of the centroid method and the HA method were not significantly different $(p>0.05)$. The mean distance from each individual to the mean rotation centre of the cylinder, centroid, conventional and helical axis method were 19, 18, 16 and $21 \mathrm{~mm}$ respectively. The mean distance from the HA method to the cylinder, centroid and conventional method were 43.0, 48.6 and $46.3 \mathrm{~mm}$ respectively. In Table 2 and figure 5, the mean location of the rotation centre per method is shown. 
Table 2. Mean ( $\pm 1 S D$ ) of the shoulder complex rotation centre for four different methods.

\begin{tabular}{llll}
\hline Method & $\mathrm{X}(\mathrm{mm})$ & $\mathrm{Y}(\mathrm{mm})$ & $\mathrm{Z}(\mathrm{mm})$ \\
\hline Cylinder & $-83.4(11.4)^{\star \star}$ & $-0.1(16.7)^{\star \star}$ & $165.0(5.6)^{\star \star}$ \\
Centroid & $-93.0(13.1)^{\star \star}$ & $-18.1(13.5)(\mathrm{ns})$ & $173.6(7.0)^{\star \star}$ \\
Conventional & $-75.4(10.3)(\mathrm{ns})$ & $15.2(14.1)^{\star *}$ & $156.8(5.2)^{\star *}$ \\
Helical axes & $-63.5(16.8)$ & $-21.5(13.2)$ & $139.8(11.1)$ \\
linder, centroid and conventional were compared to the reference (helical axes). ${ }^{* *}=p<0.01 ; n s=p>0.05$.
\end{tabular}

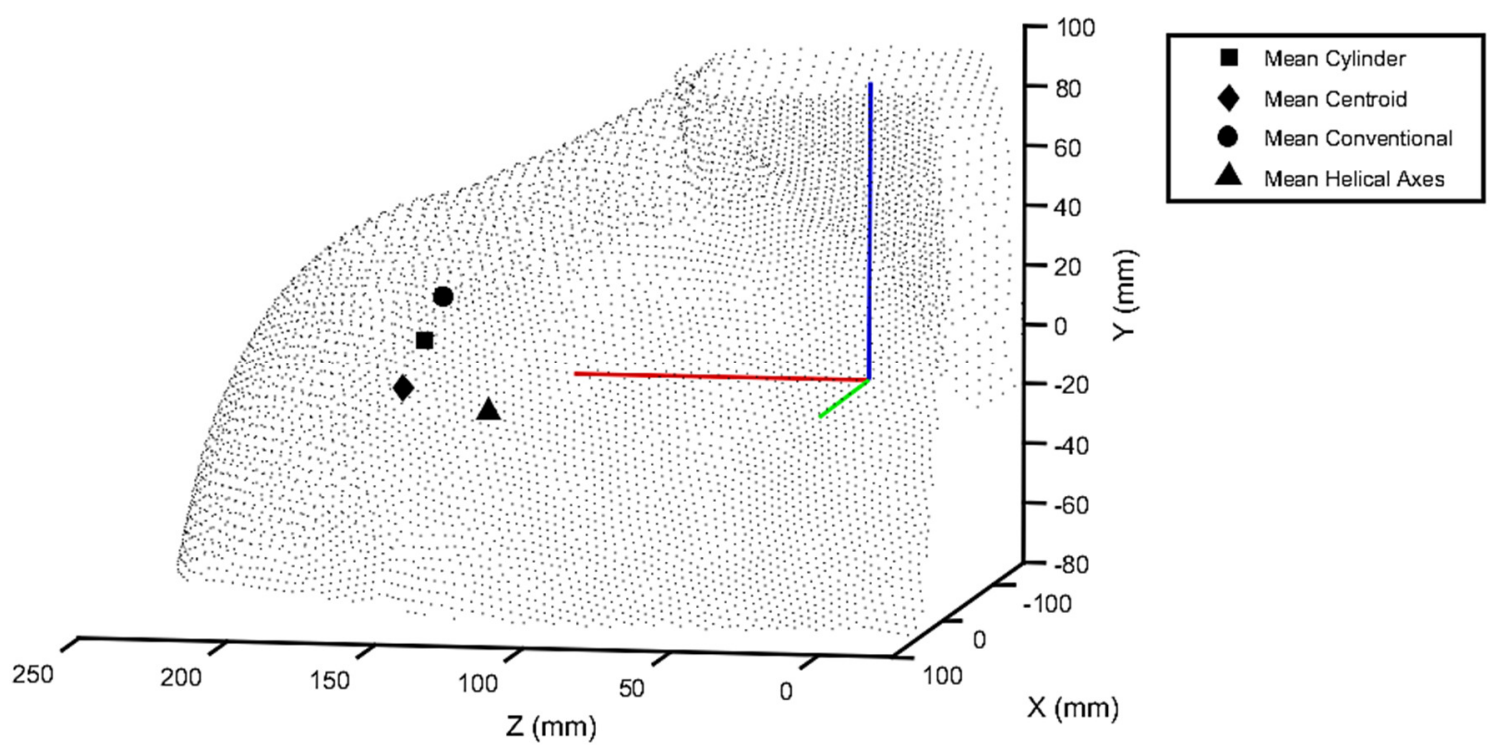

Fig. 5. The mean coordinates of the shoulder complex rotation centre for four methods in frontal view: the cylinder method (square), centroid method (diamond), conventional method (circle) and helical axes method using Optotrak (triangle). For interpretation, the point cloud of PP_014 is plotted. X-(green), Y- (blue) and Z-axis (red) are shown as lines.

\section{Discussion}

For the design of the exoskeleton a single rotation point was required. In this study, two methods were developed and analysed to calculate the shoulder complex rotation centre from 3D body scans: the cylinder and centroid method. Together with the conventional method, the 3D scan derived rotation centres were compared to the rotation centre determined using the HA method. It was hypothesized that calculation of the shoulder complex rotation centre using 3D body scan methods obtained the same result as calculated from the HA method and that 3D body scan methods result in an estimate of the rotation centre closer to the HA method in comparison to the conventional method. The HA method was used as a reference to validate the 3D body scan derived methods. Although the HA method is validated for the glenohumeral joint, this does not mean that it is also valid for a complex such as the shoulder [8], [11], [13]-[15], [20]. In the first $30^{\circ}$ to $60^{\circ}$ of arm elevation, the major part of the motion is occurring from the glenohumeral joint since the scapula is in the setting phase [38]. Scapular motion in the setting phase is highly variable between individuals, ranging from a few degrees of scapular motion to no motion at all. After $50^{\circ}$, the glenohumeral joint and scapulothoracic joint both contribute in a linear way to the motion in a 1.7:1 ratio [39]. In this study, the motion was performed until $90^{\circ}$ elevation, which means that the motion is mainly occurring from the glenohumeral joint but the contribution of the scapula should not be omitted. Therefore, it is plausible that the HA method will be valid for the shoulder complex. Papers of Amabile et al. and Doorenbosch et al. are the only ones to discuss the rotation centre of the shoulder complex and both used the HA method to find this point [12], [18]. Although the HA method was used in our study as a reference method, the best best estimate of a rotation centre of the shoulder complex under the assumption that there is a single point is not known. To our knowledge, there are no studies conducted with X-ray or CT scans to find the shoulder complex rotation centre. Both studies [12], [18] showed that there was 
considerable variation between the participants, which is in line with our observations. In the study of Amabile et al. [18], the largest rotation centre standard deviation was $16 \mathrm{~mm}$. The largest standard deviation in our study was $17 \mathrm{~mm}$. Doorenbosch et al. [12] reported slightly lower values, their maximal value of the location of the shoulder rotation centre standard deviation was $8 \mathrm{~mm}$. However, they only included 7 subjects in their research while 13 subjects were included in this study. Our larger standard deviations can be partly attributed to differences between participants. This also stresses the idea of personalized design of the exoskeleton. Further comparison of these papers to our research is difficult since Doorenbosch et al. used a different coordinate system with the origin in AA [12]. Amabile et al. used the ISB recommended coordinate system, but included different bony landmarks whereby it was only possible to estimate the X-and Z-coordinate [18], [20]. In comparison to our estimates, their rotation centre was more medial and dorsal. Another difference is that these studies calculated a location of the rotation centre for a specific plane and motion. We did not divide the motions but combined them to compute a total average rotation centre in three dimensions. Furthermore, some measurement errors can influence the results of the HA calculations. It is known that this method is sensitive for low velocities, therefore a cut-off of $10 \%$ of the maximal rotational velocity is used. Also, the use of cluster markers can be a source of error. The cluster marker can shift due to the movement of the arm and alter the trajectory. In that case, it is better to use 3D scanning since this uses the whole arm as a large collection of marker points. Since we want to give a conclusion about the accuracy of the rotation centre estimates from our 3D scanning methods, HA can be used as the reference, keeping in mind that this method is not per definition the best estimate of the single rotation point that is required for the exoskeleton design. Results showed that a nonsignificant difference for the Y-coordinate of the centroid method was found. Looking at the data, it was observed that the variation between methods (3D derived methods versus $\mathrm{HA}$ ) exceeded the individual variation within a method (see figure 6).

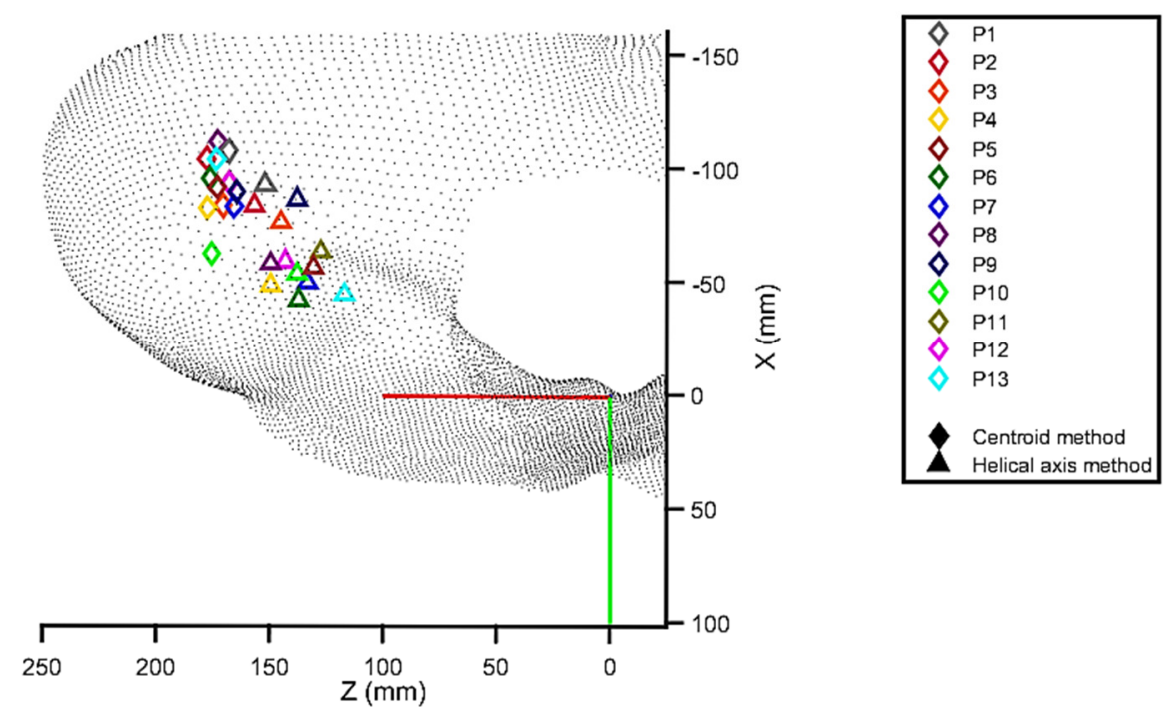

Fig. 6. The coordinates of the shoulder complex rotation centre for the centroid method and the helical axes method are shown in a transverse view. It can be seen that the differences between the coordinates within a group are smaller than the difference between the groups within one person. X- (green), Z-axis (red) are shown as lines.

The variation between the methods can indicate a systematic error. It was investigated if a correction factor can be applied to the data. If $30 \mathrm{~mm}$ is added to the X-and Z-coordinate of the centroid method, there are no significant differences anymore between the centroid method and the HA method. The Euclidean distance for every individual between the HA method and the centroid method was calculated before and after the correction. Due to the correction, the individual rotation centres became on average $24.9 \mathrm{~mm}$ closer to the HA rotation centres. On average, the distances between the HA rotation centres and centroid rotation centres after correction were $23.8 \mathrm{~mm}$. A correction could also be applied to the conventional method, although this correction resulted in a less desired result where the rotation centre became $22.4 \mathrm{~mm}$ closer after the correction. A correction factor removes the systematic error, but after correction, the distance between the $3 \mathrm{D}$ body scan methods is not within $10 \mathrm{~mm}$ of the HA rotation centre [8], [15], [17]. Since this was the first step towards the use of $3 \mathrm{D}$ body scanning for biomechanical purposes, we believe that changes in the methods could 
eventually lead to better results. For instance, performing the measurements at the same location could already lead to more precise results. The same bony landmarks were palpated on different days. The low reproducibility rate of spinal palpation is reported in the literature [40]. Furthermore, the bony landmarks were manually selected in Meshlab. To determine the accuracy of point picking, the four most important bony landmarks ST, XP, C7 and T6 (which construct the coordinate system) are determined 10 times in the same 3D body scan. ST showed the largest deviation in point pick accuracy of [ $\left[\begin{array}{lll}1.9 & 1.8 & 1.1\end{array}\right] \mathrm{mm}$ in X-, Y- and Z-direction respectively.

A few assumptions were made during this research which should be addressed. For instance, the humerus is not a straight, uniform bone. The proximal part of the shaft is somewhat larger than the distal part. Due to the large muscles, biceps and triceps, there could be variation in the exact position of the humerus in the upper arm. Furthermore, the arm is not a true cylinder. The proximal part of the upper arm has a larger circumference than the distal part. Other studies also fitted a cylinder around the proximal shaft of the humerus to define an axis for a reference frame [33], [34]. Roberts and Foley (1991) stated that a cylinder was the best reproducible geometric model even though there was a deviation of $11 \%$ between the point cloud and the fitted model [34]. To overcome this problem, the centroid method was proposed in our research. The results of these two methods were significantly different. In the proposed methods, a few constraints were required for the fitting algorithm. A reference vector was created which we defined to be the line between the midpoint of the elbow and the rotation centre calculation in the conventional way. This reference vector was used in the algorithm to start the search for the best fit of the cylinder. The number of fitted cylinders was also determined by a sensitivity analysis where the number of fitted cylinders was varied. The mean of the distances between the midpoints of the shortest distance lines and the rotation centre is called the error. A lower error means the midlines deviate from each other less. Results showed that at 250 cylinders, the addition of more cylinders does not influence the outcome (see figure 7).

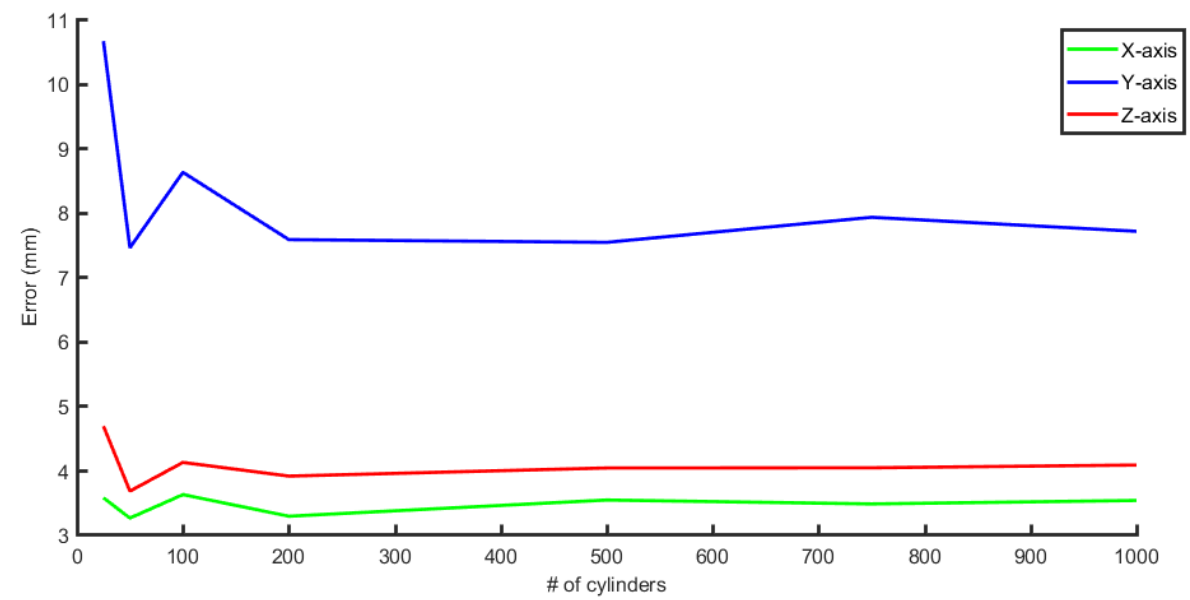

Fig. 7. The error is plotted against the number of fitted cylinders. The error is the mean of the distances from the midpoints of the shortest distance lines to the rotation centre. With a small number of cylinders, the error is large. After 200 cylinders, there is less variation in the error.

Another point of concern is that the algorithm could encounter problems with obese subjects, which is often the case with Duchenne patients. They have an excessive amount skin and fat on the upper arm which could affect the algorithm. The algorithm would not be able to fit a cylinder through the point cloud of the 3D body scan. The centroid method could still be used, but the midline through the upper arm would probably not coincide with the humerus bone. In this study, the goal was to develop the method and algorithm. Future research can compare 3D body scans of skinny and obese participants and investigate how large the effect is on the calculation of the rotation centre, due to different forms of the arm. Another topic of interest is finding the shoulder complex rotation centre in vivo. CT scanning can provide the solution to our uncertain reference of the HA method. From a CT scan, both the mid-line of the humerus bone and mid-line of the arm can be calculated. We performed a preliminary study on a cadaveric body to determine the minimal radiation exposure for quality images. An experiment was performed where the radiation dose was lowered gradually and the precision of motion parameters needed for rotation axes estimation was analysed. This resulted in a reduction of the radiation by $76 \%$. Future research can elaborate on this preliminary study by making 3D CT scans in different postures and compare them to calculate a shoulder complex rotation centre. 


\section{Conclusions}

We demonstrated that it is possible to calculate a rotation centre using 3D body scanning, but the four methods differed considerably. To remove the systematic error between methods, a correction factor of $30 \mathrm{~mm}$ in the X-and Z-direction was applied to the centroid method that was preferred over the cylinder method. With this correction factor, the individual 3D body scan rotation centres were on average $23.8 \mathrm{~mm}$ away from the individual HA rotation centres, bearing in mind that the HA is also an estimate and this method is not verified within the body for the shoulder complex. X-ray imaging should be used as the golden standard. The random error of the 3D body scanning methods did not exceed the random error of the HA method, suggesting that 3D body scanning can be used for biomechanical modelling. 3D body scanning offers a simple method to collect anthropometric data and possibly biomechanical data about a subject, but methods need to be refined.

\section{Acknowledgements}

We would like to thank all the participants who invested their time in this research project. We acknowledge the contribution of W. Schallig, L. Verweij and I. Dobbe to this study.

\section{References}

[1] B. Bru and V. Pasqui, 'Localisation of the Instantaneous Axis of Rotation in Human Joints', 2010 [Online]. Available: 10.1007/978-90-481-9262-5.

[2] S. Holzreiter, 'Calculation of the instantaneous centre of rotation for a rigid body', J. Biomech., vol. 24, no. 7, pp. 643-647, 1991.

[3] B. Tondu, 'Estimating shoulder-complex mobility', Appl. Bionics Biomech., vol. 4, no. 1, pp. 19-29, 2007 [Online]. Available: 10.1080/11762320701403922.

[4] F. Van der Helm et al., 'Geometry parameters for musculoskeletal modelling of the shoulder system', J. Biomech., vol. 25, no. 2, pp. 129-144, Feb. 1992 [Online]. Available: 10.1016/0021-9290(92)90270-B.

[5] J. K. Udupa and G. T. Herman, 3D Imaging in Medicine, Second Edition. CRC Press, 1999.

[6] J. Victor et al., 'How precise can bony landmarks be determined on a CT scan of the knee?', Knee, vol. 16, no. 5, pp. 358-365, 2009 [Online]. Available: 10.1016/j.knee.2009.01.001.

[7] H. Kainz et al., 'Estimation of the hip joint centre in human motion analysis: A systematic review', Clin. Biomech., vol. 30, no. 4, pp. 319-329, 2015 [Online]. Available: 10.1016/j.clinbiomech.2015.02.005.

[8] B. Michaud et al., 'Determining in vivo sternoclavicular, acromioclavicular and glenohumeral joint centre locations from skin markers, CT-scans and intracortical pins: A comparison study', Med. Eng. Phys., vol. 38, no. 3, pp. 290-296, Mar. 2016 [Online]. Available: 10.1016/j.medengphy.2015.12.004.

[9] M. L. Hull, 'A Finite Element Model of the Human Knee Joint for the Study of Tibio-Femoral Contact', J. Biomech. Eng., vol. 124, no. 3, p. 273, 2002 [Online]. Available: 10.1115/1.1470171.

[10] R. M. Ehrig et al., 'A survey of formal methods for determining the centre of rotation of ball joints', J. Biomech., vol. 39, no. 15, pp. 2798-2809, Jan. 2006 [Online]. Available: 10.1016/j.jbiomech.2005.10.002.

[11] H. E. J. Veeger, 'The position of the rotation center of the glenohumeral joint', J. Biomech., vol. 33, no. January 2001, pp. 1711-1715, 2000.

[12] C. A. Doorenbosch et al., 'Determination of functional rotation axes during elevation of the shoulder complex.', J. Orthop. Sports Phys. Ther., vol. 31, no. 3, pp. 133-7, Mar. 2001 [Online]. Available: 10.2519/jospt.2001.31.3.133.

[13] M. Stokdijk et al., 'The glenohumeral joint rotation centre in vivo', J. Biomech., vol. 33, no. 12, pp. 1629-1636, 2000.

[14] T. Monnet et al., 'Comparison of the SCoRE and HA methods for locating in vivo the glenohumeral joint centre', J. Biomech., vol. 40, no. 15, pp. 3487-3492, 2007.

[15] A. A. Nikooyan et al., 'Comparison of Two Methods for In Vivo Estimation of the Glenohumeral Joint Rotation Center ( GH-JRC) of the Patients with Shoulder Hemiarthroplasty', PLoS One, vol. 6, no. 3, pp. 1-7, 2011 [Online]. Available: 10.1371/journal.pone.0018488.

[16] B. A. MacWilliams, 'A comparison of four functional methods to determine centers and axes of rotations', Gait Posture, vol. 28, no. 4, pp. 673-679, 2008 [Online]. Available: 10.1016/j.gaitpost.2008.05.010. 
[17] M. Lempereur et al., 'In vivo estimation of the glenohumeral joint centre by functional methods: Accuracy and repeatability assessment', J. Biomech., vol. 43, no. 2, pp. 370-374, Jan. 2010 [Online]. Available: 10.1016/j.jbiomech.2009.09.029.

[18] C. Amabile et al., 'The centre of rotation of the shoulder complex and the effect of normalisation', J. Biomech., vol. 49, no. 9, pp. 1938-1943, 2016 [Online]. Available: 10.1016/j.jbiomech.2016.03.035.

[19] C. W. Spoor and F. E. Veldpaus, 'Rigid body motion calculated from spatial coordinates of markers', J. Biomech., vol. 13, no. 4, pp. 391-393, 1980 [Online]. Available: 10.1016/00219290(80)90020-2.

[20] G. Wu et al., 'ISB recommendation on definitions of joint coordinate systems of various joints for the reporting of human joint motion-Part II: shoulder, elbow, wrist and hand', J. Biomech., vol. 38, no. 5, pp. 981-992, May 2005 [Online]. Available: 10.1016/j.jbiomech.2004.05.042.

[21] H. A. M. Daanen and F. B. Ter Haar, '3D whole body scanners revisited', Displays, vol. 34, no. 4, pp. 270-275, Oct. 2013 [Online]. Available: 10.1016/j.displa.2013.08.011.

[22] P. Treleaven and J. Wells, '3D Body Scanning and Healthcare Applications', Computer (Long. Beach. Calif)., vol. 40, no. 7, pp. 28-34, Jul. 2007 [Online]. Available: 10.1109/MC.2007.225.

[23] P. N. Kooren et al., 'Design and control of the Active A-Gear: A wearable 5 DOF arm exoskeleton for adults with Duchenne muscular dystrophy', Proc. IEEE RAS EMBS Int. Conf. Biomed. Robot. Biomechatronics, no. June, pp. 637-642, 2016.

[24] P. N. Kooren et al., 'Design and pilot validation of A-gear: a novel wearable dynamic arm support', J. Neuroeng. Rehabil., vol. 12, no. 1, p. 83, 2015.

[25] J. K. Mah et al., 'A systematic review and meta-analysis on the epidemiology of Duchenne and Becker muscular dystrophy.', Neuromuscul. Disord., vol. 24, no. 6, pp. 482-91, Jun. 2014 [Online]. Available: 10.1016/j.nmd.2014.03.008.

[26] P.-Y. Lin et al., 'A theoretical study of weight-balanced mechanisms for design of spring assistive mobile arm support (MAS)', Mech. Mach. Theory, vol. 61, pp. 156-167, Mar. 2013 [Online]. Available: 10.1016/j.mechmachtheory.2012.11.003.

[27] CBS. [Online]. Available: https://www.cbs.nl/nl-nl.

[28] G. Kurillo et al., 'Evaluation of upper extremity reachable workspace using Kinect camera', Technol. Heal. Care, vol. 21, no. 6, pp. 641-656, 2013 [Online]. Available: 10.3233/THC130764.

[29] CloudCompare. [Online]. Available: http://www.danielgm.net/cc/.

[30] P. Cignoni et al., 'MeshLab: an Open-Source Mesh Processing Tool', Sixth Eurographics Ital. Chapter Conf., pp. 129-136, 2008.

[31] (2013, ), Geomagic Studio. [Online]. Available: https://www.3dsystems.com/software.

[32] P. Boileau and G. Walch, 'The Three-Dimensional Geometry of the Proximal Humerus: Implications for Surgical Technique and Prosthetic Design', J. Bone Jt. Surg., vol. 79, no. 5, pp. 857-865, Sep. 1997 [Online]. Available: 10.1302/0301-620X.79B5.7579.

[33] B. A. Garner and M. G. Pandy, 'A kinematic model of the upper limb based on the visible human project (VHP) image dataset', Comput. Methods Biomech. Biomed. Engin., vol. 2, no. 2, pp. 107-124, 1999 [Online]. Available: 10.1080/10255849908907981.

[34] S. N. J. Roberts and P. J. Foley, 'The Geometry of the humeral head and the of design of prostheses', Br. Editor. Soc. Bone Jt. Surg., vol. 73, no. 4, pp. 647-650, 1991.

[35] P. H. S. Torr and A. Zisserman, 'MLESAC: A New Robust Estimator with Application to Estimating Image Geometry', Comput. Vis. Image Underst., vol. 78, no. 1, pp. 138-156, 2000.

[36] H. J. Woltring et al., 'Finite centroid and helical axis estimation from noisy landmark measurements in the study of human joint kinematics', J. Biomech., vol. 18, no. 5, pp. 379389, 1985 [Online]. Available: 10.1016/0021-9290(85)90293-3.

[37] H. J. Woltring, 'Data processing and error analysis', Biomech. Hum. Mov., pp. 203-237, 1990.

[38] V. T. Inman et al., 'Observations on the function of the shoulder joint', J. bone Jt. Surg., vol. 97, no. 38, pp. 9547-9549, 1994 [Online]. Available: 10.1525/sp.2007.54.1.23.

[39] P. W. McClure et al., 'Direct 3-dimensional measurement of scapular kinematics during dynamic movements in vivo', J. Shoulder Elb. Surg., vol. 10, no. 3, pp. 269-277, 2001.

[40] M. J. Stochkendahl et al., 'Manual examination of the spine: a systematic critical literature review of reproducibility', J. Manipulative Physiol. Ther., vol. 29, no. 6, pp. 475-485, 2006 [Online]. Available: 10.1016/j.jmpt.2006.06.011. 